\title{
Tables of Certain Functions Occurring in Dynamics of Structures
}

The following is a short description of tables contained in K. HoHENEMSER and W. Prager, Dynamik der Stabwerke. Eine Schwingungslehre für Bauingenieure Berlin, Springer, $1933 .{ }^{1}$ vi, 368 p. $15.5 \times 23.1 \mathrm{~cm}$. These tables were computed with the view to facilitating the solution of certain standard problems of Dynamics of Structures.

1. The frequency functions. The natural frequencies of the small flexural vibrations of a prismatic elastic beam which is elastically supported and elastically clamped at both ends are found from the roots $\lambda$ of the following equation: ${ }^{2}$

where

$$
\begin{aligned}
e_{0} f_{0}\left[e_{1} f_{1} D(\lambda)\right. & \left.-e_{1} A(\lambda)+f_{1} B(\lambda)+E(\lambda)\right] \\
& +e_{0}\left[e_{1} f_{1} A(\lambda)-e_{1} S(\lambda)-f_{1} C(\lambda)+A(\lambda)\right] \\
& -f_{0}\left[e_{1} f_{1} B(\lambda)+e_{1} C(\lambda)-f_{1} S(\lambda)+B(\lambda)\right] \\
& +e_{1} f_{1} E(\lambda)-e_{1} A(\lambda)+f_{1} B(\lambda)+D(\lambda)=0,
\end{aligned}
$$

$$
\begin{aligned}
& A(\lambda)=\cosh \lambda \sin \lambda+\sinh \lambda \cos \lambda, \\
& B(\lambda)=\cosh \lambda \sin \lambda-\sinh \lambda \cos \lambda, \\
& C(\lambda)=2 \cosh \lambda \sin \lambda, \\
& S(\lambda)=2 \sinh \lambda \sin \lambda, \\
& D(\lambda)=\cosh \lambda \sin \lambda-1, \\
& E(\lambda)=\cosh \lambda \cos \lambda+1
\end{aligned}
$$

The dimensionless parameters $e_{0}, e_{1}$ characterize the rigidity of the clamping and $f_{0}, f_{1}$ the rigidity of the supports, the subscripts 0 and ${ }_{1}$ referring to the two ends of the beam. The roots $\lambda$ of the equation (1) are connected with the (circular) frequency $\omega$ of the flexural vibrations by $\lambda=l \omega^{1 / 2}(\mu / E I)^{1 / 4}$, where $l$ is the length, $\mu$ the mass per unit length and $E I$ the flexural stiffness of the beam. Since any member of a plane frame consisting of straight beams may be considered as a beam elastically supported and elastically clamped at its two ends, equation (1) can be applied to find the natural frequencies of any such frame. Experience shows that in such frequency computations not only the functions (2) but also the ratios $S(\lambda) / B(\lambda)$, $C(\lambda) / A(\lambda)$, and $B(\lambda) / D(\lambda)$ occur frequently. The functions (2) as well as these ratios are tabulated, along with $1 / \lambda$ and $\cot \lambda$, p. 323-343, for $\lambda$ $=[0.00(0.02) 10.00 ; 5 \mathrm{D}]$. These tables were computed by F. W. WaltKInG and Mrs. K. HoHenemser.

2. The amplitudes of displacement and bending moment in the forced vibrations of a beam on two supports. Consider a prismatic elastic beam of length $l$ extending along the $x$-axis of a system of rectangular coordinates $x, y$ from $x=0$ to $x=l$ and resting on pin-supports at these two points. If at the end $x=0$ this beam is loaded by a periodic couple $C=C_{0} \cos \omega t$ of a given (circular) frequency the bending moment at the cross section $x$ can be computed according to

$$
M(\xi, t)=\underset{101}{C_{0} \chi(\lambda, \xi) \cos \omega t}
$$


where $\xi=x / l$, while $\lambda$ is defined in the same way as above and

$$
\chi(\lambda, \xi)=\frac{\sinh \lambda(1-\xi)}{2 \sinh \lambda}+\frac{\sin \lambda(1-\xi)}{2 \sin \lambda} \text {. }
$$

The deflection at the cross section $x$ is given by

where

$$
y(\xi, t)=\frac{C_{0}}{\omega} V \mu E I \bar{\chi}(\lambda, \xi),
$$

$$
\bar{\chi}(\lambda, \xi)=-\frac{\sinh \lambda(1-\xi)}{2 \sinh \lambda}+\frac{\sin \lambda(1-\xi)}{2 \sin \lambda} .
$$

The functions (3) and (4) are tabulated, to $4 D$ (p. 344-353) for $\lambda=0.0(0.1)$ 10.0 , and $\xi=0.0(0.1) 1.0$. These tables were computed by W. Prager.

3. Tables for the coefficients appearing in the four moment equation of structural dynamics. An important problem of structural dynamics is to determine the displacements, shear forces and bending moments of the members of a plane frame which is loaded by periodic forces of the type $F=F_{0} \cos \omega t$. Once the displacements and bending moments at the two ends of a member are found the displacement and bending moments at other points of this member can be computed by means of the tables discussed in section 2. For the determination of the displacements and bending moments at the endpoints of all members of the frame equations have been developed ${ }^{3}$ which correspond entirely to the four moment equations of structural statics. Whereas in the case of statics coefficients appearing in these equations depend only on the dimensions of the structure and on the loads applied to it, in the case of dynamics these coefficients depend also on the frequency of the periodic loads. It can be shown that all these coefficients may be expressed in terms of the following functions:

$$
\begin{array}{ll}
\Phi(\lambda)=-\frac{1}{4 \lambda^{3}}\left(\tanh \frac{\lambda}{2}-\tan \frac{\lambda}{2}\right), & \bar{\Phi}(\lambda)=\frac{1}{4 \lambda}\left(\tanh \frac{\lambda}{2}+\tan \frac{\lambda}{2}\right), \\
\Psi(\lambda)=-\frac{1}{4 \lambda^{2}}\left(\operatorname{sech} \frac{\lambda}{2}-\sec \frac{\lambda}{2}\right), & \bar{\Psi}(\lambda)=\frac{1}{4}\left(\operatorname{sech} \frac{\lambda}{2}+\sec \frac{\lambda}{2}\right), \\
\varphi(\lambda)=-\frac{1}{2 \lambda}(\operatorname{coth} \lambda-\cot \lambda), & \bar{\varphi}(\lambda)=\frac{\lambda}{2}(\operatorname{coth} \lambda+\cot \lambda), \\
\psi(\lambda)=-\frac{1}{2 \lambda}(\operatorname{csch} \lambda-\csc \lambda), & \bar{\psi}(\lambda)=\frac{\lambda}{2}(\operatorname{csch} \lambda+\csc \lambda) .
\end{array}
$$

These functions are tabulated, along with $\tan (\lambda / 2), \sec (\lambda / 2), \cot \lambda$, and $\csc \lambda$, for $\lambda=[0.00(0.05) 10.00 ; 5 \mathrm{D}]$. The tables were computed by $\mathrm{S}$. GRADSTEIN.

\section{Brown University}

W. Prager

1 Of this work there was a Ukrainian translation, Dinamika Sporudzhen 2 , KharkovKiev, Naukovo-Tekhnichne Vidavnitstvo Ukraǐni, 1935, 381 p.

'See W. Prager, "Zur Berechnung der Eigenschwingungszahlen ebener und räumlicher Stabwerke," Z. techn. Physik, v. 10, 1929, p. 275-280; F. W. Waltking, "Zur Ermittlung der Eigenschwingungszahlen ebener Stabwerke," Ingenieur-Archiv, v. 2, 1931, p. 247-274. 
F. Reinitzhuber, "Beitrag zur Berechnung der Eigenschwingungszahlen räumlicher Stabwerke," idem, v. 8, 1937, p. 349-363.

${ }^{3}$ W. Prager, "Die Beanspruchung von Tragwerken durch schwingenden Lasten," Ingenieur-Archiv, v. 1, 1930, p. 527-532; S. Gradstein and W. Prager, "Beanspruchung und Formänderung von Stabwerken bei erzwungenen Schwingungen," Ingenieur-Archiv, v. 2, 1931, p. 622-650; "Berichtigungen," v. 3, 1932, p. 434. Tables of $\tan \lambda / 2, \cot \lambda, \csc \lambda$, $\sec \lambda / 2$, are given on p. $643-646$; and those for the last group of functions listed above, p. 647-650. See also E. Fliegel, "Die Elasticitätsgleichungen zweiter Art der Stabwerksdynamik," idem, v. 9, 1938, p. 20-28, where $\phi /\left(\phi^{2}+\psi^{2}\right), \psi /\left(\phi^{2}-\psi^{2}\right), 1 /(\phi-\psi)$, and $1 / \phi$ are tabulated for $\lambda=[0.00(0.50) 5.00 ; 2 \mathrm{D}]$; and R. E. Gaskell, "On moment balancing in structural dynamics," Ouarterly of Applied Mathematics, Brown University, v. 1, 1943, p. 237-249.

\section{RECENT MATHEMATICAL TABLES}

108[K].-W. G. Cochran, "The distribution of the largest of a set of estimated variances as a fraction of their total," Annals of Eugenics, v. 11, 1941, p. $47-52$. $20.2 \times 27.5 \mathrm{~cm}$.

In this paper the distribution of

$$
g=\left(\text { largest } u_{i}\right) / \sum_{i=1}^{n} u_{i}
$$

is studied for the case where $u_{1}, u_{2}, \cdots, u_{i}, \cdots, u_{n}$ are mutually independent random variables each distributed as $\chi^{2} \sigma^{2}$ for $r$ degrees of freedom, i.e.,

$$
p\left(u_{i}\right) d u_{i}=\frac{u_{i}^{(r-2) / 2} e^{-u_{i} / 2 \sigma^{2}}}{\left(2 \sigma^{2}\right)^{r / 2} \Gamma(r / 2)} d u_{i}, \quad(i=1,2, \cdots, n) .
$$

The values of $g$ which have a probability 0.05 of being exceeded in random sampling are tabulated for $n=[3(1) 10 ; 4 \mathrm{D}], r=1(1) 6(2) 10$.

The utility of these " $5 \%$ points of $g$ " derives from the fact that $u$ may be interpreted as the "sum of squares with $r$ degrees of freedom" corresponding to an unbiased estimate, $s^{2}=u / r$, of the variance, $\sigma^{2}$, of a Gaussian population. In consequence, the distribution of $g$ provides the distribution stated in the title, and the " $5 \%$ points of $g$ " provide a test of whether a variance estimate which appears to be anomalously larger than the others of a set is an estimate of the same $\sigma^{2}$.

The distribution of $g$ for the case of $r=2$ was obtained by R. A. Fisher, "Tests of significance in harmonic analysis," R. So. London, Proc. v. 125A, 1929, p. 180-190, who proposed its use in the harmonic analysis of a series to test the statistical significance of a particular term which is picked out on inspection because of its exceptional magnitude. In this paper Fisher gives a table of the 5\% points of $g$ to $5 \mathrm{D}$ for $r=2$ and $n=5(5) 50$. In R. A. Fisher, "The sampling distribution of some statistics obtained from non-linear equations," Annals of Eugenics, v. 9, 1939, p. 238-249, the distribution of $g$ is employed to illustrate the disturbance introduced into the analysis of variance test of a regression equation when the equation is 'non-linear' in the sense that the eliminant of the regression coefficients is a non-linear function of the observations which satisfy the regression equation exactly. W. L. Stevens, "Solution to a geometrical problem in probability," Annals of Eugenics, v. 9, 1939 , p. 315-320, found that the distribution of $g$ for $r=2$ provides the answer to the following question: "On the circumference of a circle of unit length, $n$ arcs, each of length $x$, are marked off at random. What is the probability that every point of the circle is included in at least one of the arcs?" This curious coincidence is explained by R. A. Fisher, "On the similarity of the distributions found for the test of significance in harmonic analysis, and in Stevens' problem in geometrical probability," Annals of Eugenics, v. 10, 1940, p. 14-17, and the distribution of the second largest ratio, $g_{2}$, when $r=2$ is also discussed. This paper is followed, p. 17, by W. L. Stevens, "A table of the test of significance in harmonic analysis," in which the $5 \%$ points of $g_{1}$ (our $g$ ) and $g_{2}$ are tabulated for $r=2$ and $n=[3(1) 10(5) 50 ; 5 \mathrm{D}]$. 